\title{
Veranlassung und Zielsetzung
}

Städte als bevorzugte Lebensräume stehen in Zeiten des Klimawandels, der Urbanisierung sowie des demographischen Wandels zunehmend vor der Herausforderung neuer Systembelastungen. Der Wasser- sowie Energiehaushalt ist durch die zunehmende Versiegelung von starken Veränderungen betroffen. In Siedlungsgebieten ist von einer Verringerung der Grundwasserneubildung und Verdunstung auszugehen, während Abflussvolumen und Abflussspitzen zunehmen und der Niederschlagsabfluss stark beschleunigt auftritt. Nachhaltige Anpassungsstrategien sollen diesen negativen Effekten entgegenwirken.

Im Sinne einer integralen Entwässerungsplanung ist es das Ziel, den lokalen Wasserhaushalt hydraulisch sowie stofflich möglichst wenig zu beeinträchtigen (DWA-A 100 2006). Aus Sicht der Stadthydrologie stellt die Verdunstung einen entscheidenden Parameter in der Wasserhaushaltsbilanzierung dar. Dieser wird von verschiedenen meteorologischen und hydrologischen Einflüssen geprägt und steht gleichermaßen in Abhängigkeit von Standortfaktoren wie Bodeneigenschaften und Vegetation.

Im Rahmen der ressourceneffizienten Stadtentwicklung wurden in der Vergangenheit für die Verdunstung oftmals nur überschlägige Ansätze gewählt, die mit gemittelten Gebietseigenschaften in die Wasserhaushaltsmodellierung eingingen. Kleinskalige Strukturen, wie die verschiedenen urbanen Vegetationsflächen, fanden in nur sehr geringem Maße Abbildung in den Modellen, obwohl die nachhaltige Strategieentwicklung auf dieser Ebene abläuft.

Ziel dieser Arbeit ist die Weiterentwicklung der Verdunstungsmodellierung, die im Niederschlag-Abfluss Modell SWMM für die Abbildung blau-grüner Infrastruktur angewendet wird. Dabei sollen so weit wie möglich die komplexen Zusammenhänge des Systems Pflanze-Boden-Atmosphäre beachtet werden, so dass eine realitätsnahe Modellierung verschiedener Vegetationselemente möglich ist. Gründende Basis ist eine umfassende Literaturrecherche zur Verdunstungsmodellierung von Vegetationsflächen. Für die auszuwählenden Ansätze sollen Erkenntnisse hinsichtlich Parametersensitivitäten gewonnen und darauf aufbauend Empfehlungen zur Parametrisierung gegeben werden. Die Übertragung der Modellierung auf größerskalige Gebiete soll die Anwendbarkeit für durchgehende Planungen im Sinne des „water sensitive urban design“ sichern. 